Revista del Centro de Investigación de la Universidad La Salle

Vol. 14, No. 53, Enero-Junio, 2020: 181-210

DOI: http://doi.org/10.26457/recein.v14i53.2687

\title{
El derecho a la información de grupos susceptibles de discriminación en la comunicación social del Gobierno Federal mexicano ante la pandemia por SARS-CoV-2 \\ The Right to Information of vulnerable groups to be disciminated against, of the Mexican Federal Government's social communication network, in the face of the SARS CoV-2 pandemic.
}

\author{
Ricardo Bernal Lugo ${ }^{1}$ \\ Universidad La Salle México(México) \\ Claudia Ivette Pedraza Bucio \\ Universidad La Salle México (México) \\ Leonardo Jiménez Loza \\ Universidad La Salle México (México) \\ Jorge Valtierra Zamudio \\ Universidad La Salle México (México)
}

Recibido: 06 de junio de 2020

Aceptado: 21 de agosto de 2020

Publicado: 21 de septiembre de 2020

\section{Resumen}

El presente trabajo traza los primeros resultados de un estudio orientado a valorar los límites y alcances de la parte más visible de la estrategia de comunicación social del Gobierno

\footnotetext{
${ }^{1}$ Email: ricardo.bernal@lasalle.mx
}

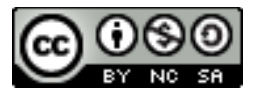

Revista del Centro de Investigación. Universidad La Salle por Dirección de Posgrado e Investigación. Universidad La Salle Ciudad de México se distribuye bajo una Licencia Creative Commons Atribución- 
Federal en el contexto de la pandemia por SARS-CoV-2 en lo que corresponde a la garantía de una de las tres dimensiones del derecho a la información, a saber, el derecho a ser informado, referido específicamente a grupos susceptibles de discriminación. Después de argumentar que el derecho a ser informado debe contemplar la difusión de contenidos diferenciados para grupos sociales distintos, particularmente para aquellos susceptibles de discriminación, estudiamos la prioridad informativa que desde la comunicación gubernamental se otorgó a los sectores de la población susceptibles a ser discriminados durante la contingencia. El método utilizado fue el análisis de contenido, para lo cual se definieron los siguientes indicadores: la frecuencia con que estos grupos fueron mencionados en la conferencia vespertina de la Secretaria de Salud, el tipo de información que se ofreció respecto a ellos y el lugar desde el que fueron enunciados. Los resultados obtenidos muestran que los grupos susceptibles de discriminación sí fueron contemplados en la conferencia, pero que hay importantes diferencias respecto a la prioridad de unos grupos sobre otros, y que no en todos los casos la información resulta pertinente para los riesgos específicos derivados de la pandemia para estos grupos.

Palabras clave: SARS-CoV-2; Derecho a la información; Derecho a la Igualdad y no discriminación; comunicación social; información pública; Gobierno de México. 


\section{Abstract}

This paper lays out the first results of a study aimed at assessing the limits and scope of the most visible part of the Governmental social communication strategy in the context of the SARS-CoV-2 pandemic concerning the guarantee of one of the three dimensions of the right to information, namely, the right to be informed that vulnerable groups to discrimination have. After arguing that the right to information should contemplate the dissemination of differentiated content for different social groups, particularly for those susceptible to discrimination, we studied the informational priority that government communication granted to those sectors of the population (those discriminated against) during the pandemic contingency. The method we used is Content Analysis of the following indicators: How often are mentioned these groups during the Secretary of Health Evening Conference, the kind of information offered regarding them, and the place from which enunciate them. The result of this research shows that during the conferences of the Secretary of Health authorities, they do consider those vulnerable groups to discrimination. However, there are differences concerning the priority some groups have over others. Hence, not in all cases, the information is relevant to the specific risks derived from the pandemic for these groups.

Keywords: SARS-CoV-2; Right to Information; Right to Equality and Non-Discrimination; Social Communication; Public Information; Government of Mexico. 


\section{Introducción}

En el siguiente texto presentamos los resultados de la primera fase de una investigación orientada a estudiar la manera en la que se ha atendido el derecho a ser informado de grupos susceptibles de discriminación en el contexto de la pandemia por SARS-CoV-2. Los resultados aquí presentados fueron obtenidos a partir del análisis de la estrategia de comunicación social de la Secretaría de Salud del Gobierno Federal durante la pandemia recién señalada. Para ello tomamos como objeto de estudio las conferencias diarias protagonizadas por el Dr. Hugo López-Gatell Ramírez, subsecretario de Salud y encargado de la estrategia de comunicación del Gobierno Federal ante la emergencia sanitaria, debido a su alcance y al hecho de que en ellas se presenta la postura oficial del Gobierno mexicano.

Nuestro objetivo central en esta primera fase de la investigación es identificar si en lo que corresponde a grupos susceptibles de discriminación se ofrecen los elementos que permiten cumplir los requisitos mínimos para materializar una de las tres dimensiones del derecho a la información, esto es, el derecho a ser informado. Como mostraremos más adelante, el derecho a la información es un derecho complejo con características difíciles de interpretar. Sin embargo, en este estudio nos enfocaremos en la visibilización de grupos que requieren información diferenciada debido a los riesgos particulares a los que se enfrentan, por un lado, y, por el otro, en el hecho de que la información vinculada a los mismos esté relacionada con esos riesgos particulares porque consideramos que son requisitos indispensables, aunque no suficientes, para la materialización del derecho a ser informado de los grupos susceptibles de discriminación.

Con base en esta consideración, y tomando en cuenta que nuestro análisis tiene por objeto una serie de conferencias de prensa en las que fundamentalmente existen dos lugares de enunciación (el de la autoridad y el de la prensa), definimos tres criterios para la construcción de un Índice de Prioridad Informativa (IPI), a saber: 1) la inclusión o exclusión de grupos susceptibles de discriminación en el discurso gubernamental; 2) el tipo de contenido de esas menciones a partir de la vinculación o no de las mismas con los riesgos asociados a los grupos susceptibles de discriminación (información focalizada si contempla esos riesgos/información general si no los contempla); y 3) el lugar de enunciación (autoridad/prensa). 
La distinción entre información general e información focalizada se sostiene en la consideración de que el cumplimiento del derecho a ser informado implica el reconocimiento de la pluralidad de la sociedad, lo que desde nuestra perspectiva justifica la necesidad de información diferenciada en función de las características socioculturales y en atención a las desigualdades y desventajas sociales tal como se argumentará a lo largo de este trabajo.

En tanto que el derecho a la información y el derecho a la igualdad y no discriminación emanan de la Declaración Universal de los Derechos Humanos (DDH) y están consagrados en la Constitución Política de los Estados Unidos Mexicanos (CPEUM) su garantía es una obligación del Estado mexicano. De ahí la relevancia de este trabajo, ya que ayuda a identificar los avances y limitaciones en la estrategia de comunicación social de la Secretaria de Salud respecto a dos derechos constitucionales; esto, en aras de promover modificaciones específicas que favorezcan la materialización plena de los mismos no sólo en el contexto de la pandemia, sino en otros ámbitos de la vida pública.

Para desarrollar nuestro argumento se ha procedido, en un primer momento, con la explicación acerca de cómo la información ha terminado por representar un problema en el contexto de la pandemia por SARS-CoV-2. En un segundo momento explicaremos la importancia del derecho a la información, sus componentes y su relación con el derecho a la igualdad y no discriminación. A partir de ambos elementos teóricos, hemos realizado un instrumento ex profeso para el análisis de la parte más visible de la estrategia federal, el cual se orienta a identificar si se presentan elementos informativos dirigidos específicamente a grupos susceptibles de discriminación que favorezcan el cumplimiento del derecho a ser informados. En la tercera parte de este trabajo, daremos cuenta de los resultados obtenidos al utilizar este instrumento mediante lo que denominamos IPI. Por último, añadiremos nuestras conclusiones y reflexiones finales.

\section{La importancia de la información ante la pandemia por SARS-CoV-2}

A diferencia de lo ocurrido en otras crisis sociales o sanitarias donde la escasez de información podría considerarse como un problema de primer orden, la actual pandemia por SARS-CoV-2 no sólo resulta excepcional por la velocidad de propagación del virus y la 
magnitud de las consecuencias sociales y económicas, sino por la enorme cantidad de información disponible ${ }^{2}$.

En principio, la abundancia informativa podría considerarse como una conquista democrático-liberal vinculada tanto a los ideales de transparencia, control de las atribuciones del Estado y respeto a las libertades de prensa y expresión (Held, 2009), como a los avances de las tecnologías de la información. Aunque sin duda esto es cierto, la pandemia por SARSCoV-2 ha mostrado con toda claridad un conjunto de problemas asociados a la sobresaturación informativa que ya eran parte de nuestras sociedades y se han acentuado en esta emergencia.

Desde la década de 1990 académicos y especialistas hablaban de un cambio en los procesos de obtención, procesamiento y difusión de información que impactaría tanto nuestras formas de interacción personal como nuestros modelos de organización social y política (Castells, 1999; Ramonet, 1998; Carr, 2010). En la última década estos cambios se han hecho mucho más presentes en ámbitos como el entretenimiento, las modalidades de participación política o nuestra relación con la prensa y con los nuevos medios de comunicación $^{3}$. Dichas transformaciones han reconfigurado nuestra vida cotidiana, abriendo posibilidades antes inéditas, por ejemplo, en nuestra relación con la prensa en donde se han logrado establecer canales de interacción en tiempo real o estrategias colectivas de respaldo o sanción. Sin embargo, también han surgido nuevos obstáculos.

Sobre este último aspecto, a principios de la década pasada el analista español Pascual Serrano (2013) ya advertía que el predominio de lo audiovisual, la tendencia cada vez más clara a la espectacularización, la sobresaturación informativa y la jibarización de la comunicación representaban un riesgo para cumplir los requisitos mínimos que el receptor

\footnotetext{
2 Para darse una idea de la magnitud de la información disponible hasta el 27 de mayo de 2020 la entrada SARS-CoV-2 en Google tenía 4, 730, 000, 000 resultados.

3 Sólo por mencionar algunos acontecimientos importantes en 2011 la plataforma Netflix, que ha revolucionado el mundo del entretenimiento, comenzó a brindar servicios fuera de los Estados unidos; la llamada Primavera árabe que puso en el centro de la ecuación a las redes sociales en las protestas políticas tuvo lugar entre 2010 y 2012; entre 2007 y 2010 los principales periódicos norteamericanos como The New York Times o USA Today comenzaron a reducir sus versiones impresas, otorgándole mayor relevancia a sus versiones digitales.
} 
de la información necesitaba ya no para valorar críticamente un hecho, sino acaso para comprender lo que se comunica.

En primera instancia la posición de Serrano parecía ser la de un "apocalíptico" retomando la ya clásica distinción de Umberto Eco entre apocalípticos e integrados (Eco, 1965)-; pero fenómenos globales como la proliferación de medios digitales creados en tiempos electorales para favorecer, denostar a figuras o formaciones políticas o crear campañas de miedo o desprestigio en redes sociales, han ilustrado la efectividad irreflexiva de la espectacularización, la jibarización y la saturación informativa deliberada.

Más recientemente, el vínculo entre información y democracia ha sido motivo de una importante polémica tanto por la acuñación del concepto de posverdad, originalmente planteado por el bloguero David Roberts y popularizado durante las elecciones norteamericanas de 2016, como por la relevancia que ha tenido el tema de la propagación de noticias falsas o fake news. Según McIntyre (2019), “el declive y fragmentación de los medios de comunicación contemporáneos, el advenimiento de las redes sociales" y "la bancarrota de los expertos", son algunas de las condiciones que explican el fenómeno de la posverdad en el que las justificaciones argumentadas y, en el peor de los casos, los planteamientos científicos son reemplazados por "hechos alternativos" mediante apelaciones emocionales que buscan reforzar las posiciones preestablecidas del público al que se dirigen.

Por su parte, las fake news, cuya historia es acaso milenaria, han encontrado un caldo de cultivo ideal en la propagación de medios digitales y en el declive del modelo de medios de comunicación que prevalecía durante la segunda mitad del siglo XX. A pesar de que, en principio, la pluralidad informativa debe considerarse como un elemento positivo para la democracia, múltiples analistas (Illades, 2018; Ramonet, 2018) han insistido en que la propagación de noticias falsas vinculadas a una agenda particular, sea política, ideológica o económica, no sólo distorsiona el debate público, sino que en el extremo puede poner en riesgo los derechos de terceros. El caso más extremo es el de los llamados "movimientos antivacunas", quienes apelan a la libertad de conciencia y de expresión para difundir información que niega la evidencia científica y que puede traducirse en decisiones sobre la salud de menores de edad. 
En suma, la espectacularización y jibarización de la información, el fenómeno de la posverdad y la propagación de fake news representan un problema en tanto que dificultan la construcción de una ciudadanía capaz de intervenir con solvencia en el debate público, anteponen al interés general los intereses particulares de grupos políticos y económicos con recursos para manipular la opinión pública (Chomsky, 2003), y, en el peor de los casos, proporcionan información que obstaculiza la consecución de derechos tan importantes como el derecho a la salud.

Ahora bien, si en circunstancias "ordinarias" el acceso oportuno a información confiable es importante, en circunstancias "extraordinarias" de emergencia sanitaria como las que han tenido lugar en todo el mundo debido a la pandemia por SARS-CoV-2 ${ }^{4}$ se vuelve crucial. No obstante, en los últimos meses la sociedad mexicana se ha visto expuesta a mensajes públicos contradictorios, a abundantes fuentes de información que se mezclan con el fenómeno de la desinformación por noticias falsas, tendenciosas y alarmistas, e incluso a llamados que desautorizan las cifras presentadas por la autoridad.

Estos fenómenos no sólo son un problema de comunicación, sino que también están relacionados con la garantía de derechos, particularmente del derecho a la información consagrado en la CPEUM. En efecto, en tanto que el acceso oportuno a información confiable y veraz es fundamental para la realización de cualquier proyecto vital de manera digna, ésta no puede depender de forma exclusiva de los medios de comunicación convencionales, de los medios digitales, de la circulación de noticias en internet o de cualquier otra vía de carácter privado, sino que debe ser concebida como un derecho $\mathrm{y}$, en tanto tal, debe ser provista por el Estado como representante institucionalizado de la sociedad en su conjunto (López-Ayllon, 2000).

Como veremos en el siguiente apartado, esto no significa de ninguna manera que el Estado pueda prohibir, limitar o restringir el derecho a la libertad de prensa o el derecho a la

\footnotetext{
${ }^{4}$ Las consecuencias globales y locales que trae consigo la situación de pandemia por SARS-CoV-2 o COVID19, no sólo se refieren al número de decesos y estragos en la salud de las sociedades. De hecho, pueden ir más allá de la crisis económica que ya se percibe en la actualidad. Economistas como Joan Martínez Alier, aluden a esta coyuntura como un momento en el que deben modificarse los modelos económicos y políticos para reducir la brecha de desigualdad social, anteponiendo la vida y el bienestar por encima del crecimiento económico (2000).
} 
libertad de expresión, sino que está obligado a garantizar que toda la población acceda a un bien público como la información en la medida en que ésta es una condición indispensable para el ejercicio de los demás derechos.

Ahora bien, como se ha documentado de manera amplia, la complejidad de una nación pluriétnica, pluricultural y plurilingüe como la mexicana; las diferencias sociales y las desigualdades históricas hacen más difícil garantizar los derechos de unas personas que los de otras (CONAPRED, 2017; COLMEX, 2018). Esto también es válido para el derecho a la información; no sólo en lo que corresponde a las dificultades en torno a su acceso, sino a la pertinencia de los formatos y los contenidos de la información.

En este orden de ideas, a las dificultades que son inherentes a los fenómenos informativos contemporáneos, debe añadirse que, en México como en muchas otras partes del mundo, las desigualdades estructurales hacen que unos grupos encuentren más dificultades para ver sus derechos materializados. De ahí que se vuelva importante centrar la atención en la manera en que se ha atendido el derecho a la información de algunos grupos específicos susceptibles de discriminación en el contexto de la pandemia por SARS-CoV-2.

En el siguiente apartado explicaremos en qué consiste el derecho a la información y cuál es su relación con el derecho a la no discriminación. Esto servirá como preámbulo teórico y conceptual para entender el instrumento de análisis propuesto y los resultados obtenidos.

\section{El Derecho a la información y el principio de igualdad y no discriminación}

\subsection{El derecho a la información}

En el artículo 19 de la Declaración Universal de los Derechos Humanos, se reconoce el derecho a la información, que implica la facultad de investigar, difundir y recibir información (Villanueva, 2006). Como parte de los derechos fundamentales, éste funciona como instrumento de protección de los bienes básicos necesarios para poder desarrollar cualquier plan de vida de manera digna.

En específico, el bien básico que protege el derecho a la información es la información pública, entendida como aquella que resulta relevante para la comunidad. En este sentido, la información pública no se limita a los datos, conocimientos y expresiones que 
se generan por cualquier institución de carácter público, sino que abarca toda aquella información que aporta al ejercicio de la ciudadanía. De hecho, el acceso a la información es una condición para que este ejercicio pueda realizarse de forma plena.

La información, como bien público, nutre la participación en los procesos que permiten el acceso a la cultura, expresarse, elegir la adscripción a grupos y comunidades específicos, recabar información y difundirla, beneficiarse de servicios públicos, acceder a mejores niveles educativos y a disfrutar otros productos de la creatividad humana; es decir, el derecho a la información es la llave para la ejecución de otros derechos. Por un lado, permite la exigibilidad de las garantías básicas para la vida digna; y, por el otro, faculta para la participación en los procesos democráticos.

Con base en lo anterior, conceptual y legalmente el acceso a la información implica tres dimensiones:

a) el derecho a atraerse información, que incluye el acceso a los archivos, registros y documentos públicos, así como la decisión de qué medio se lee, se escucha o se contempla;

b) el derecho a informar, que incluye las libertades de expresión y de imprenta, así como la constitución de sociedades y empresas informativas; y

c) el derecho a ser informado, que incluye las facultades de recibir información objetiva y oportuna, completa y con carácter universal.

La discusión de este texto se concentra en la tercera dimensión, ya que implica una garantía de información para todas las personas sin exclusión alguna; es decir, reconoce la existencia de la diversidad de los sujetos políticos de este derecho.

En México, el artículo 6to de la CPEUM establece que "el derecho a la información será garantizado por el Estado. Toda persona tiene derecho al libre acceso a información plural y oportuna, así como a buscar, recibir y difundir información e ideas de toda índole por cualquier medio de expresión". En dicho artículo, el Estado garantiza el acceso a las tecnologías, servicios de radiodifusión y telecomunicaciones como vías para hacer efectiva la última dimensión, la del derecho a ser informado. De esta enunciación constitucional interesa resaltar dos cuestiones: 
a) la responsabilidad del Estado como garante del acceso a la información;

b) la noción de información plural y oportuna.

Respecto a la primera cuestión el derecho a la información debe ser objeto de tutela y de observancia del Estado, que no sólo debe abstenerse de restringirlo, sino que tiene la obligación de generar todas las condiciones para su ejecución; es decir, se asume que el Estado evitará cualquier acción u omisión que impida la recepción de información. Además, el propio Estado debe disponer la información para los diferentes sectores de la ciudadanía. Esto último se vincula con la segunda cuestión: la garantía de la información plural.

La noción de pluralidad no sólo contempla la incorporación de diferentes puntos de vista sobre los asuntos públicos, sino que también reconoce la diversidad de la ciudadanía; es decir, se acepta la existencia de diferencias sociales, económicas, educativas, lingüísticas, culturales y de otra índole que pueden provocar que el acceso a la información sea desigual.

Estas dos cuestiones en combinación suponen la garantía de que toda la información pública anticipa la diversidad de sujetos que la necesitan como bien, sin ningún tipo de condiciones. Cuando la información no puede ser recibida o comprendida se genera una brecha informacional que, a su vez, implica una desigualdad social, ya que las personas quedan marginadas de las posibilidades de comunicación, formación, impulso económico, social y humano que ésta permite (Almenara, 2008). La existencia de brechas informacionales aumenta la desigualdad existente, limita el acceso a derechos y complica la participación política.

En adición a la disposición constitucional, otras normativas abonan a la garantía del derecho a ser informado de forma indiscriminada. Por un lado, la Ley General de Transparencia y Acceso a la Información Pública específica lo siguiente:

Art. 15. Toda persona tiene derecho de acceso a la información, sin discriminación, por motivo alguno.

Art. 13. En la generación, publicación y entrega de información se deberá garantizar que ésta sea accesible, confiable, verificable, veraz, oportuna y atenderá las necesidades del derecho de acceso a la información de toda persona. Los sujetos obligados buscarán, en todo momento, que la información generada tenga un lenguaje sencillo para cualquier persona y se 
procurará, en la medida de lo posible, su accesibilidad y traducción a lenguas indígenas.

Estas medidas armonizan con lo establecido con la Ley Federal para Prevenir y Erradicar la Discriminación, que considera como actos discriminatorios, entre otros, los siguientes:

Art. 9 XII. Bis. La falta de accesibilidad en el entorno físico, el transporte, la información, tecnología y comunicaciones, en servicios e instalaciones abiertos al público o de uso público;

XVIII. Restringir el acceso a la información, salvo en aquellos supuestos que sean establecidos por las leyes nacionales e instrumentos jurídicos internacionales aplicables.

Esta misma ley establece en su artículo 15 que cada uno de los poderes públicos federales está obligados a realizar las medidas de nivelación, inclusión y las acciones afirmativas necesarias para garantizar esto. Entre las medidas de nivelación se señalan el diseño y distribución de comunicaciones oficiales, convocatorias públicas, libros de texto, licitaciones, entre otros, en formato braille o en lenguas indígenas (Art. 15. Fracc. 3); el uso de intérpretes de lengua de señas mexicana en los eventos públicos de todas las dependencias gubernamentales y en los tiempos oficiales de televisión (Art 15. Fracc. 4); y el uso de intérpretes y traductores de lenguas indígenas (Art 15. Fracc. V).

No obstante, al revisar el marco regulatorio se hace evidente que la preocupación principal para garantizar el derecho a ser informado está en la forma: el formato accesible, el código, la lengua. Estas medidas anticipan la existencia de sujetos con barreras en el acceso a nivel formal, pero no contemplan las barreras que se pueden generar a nivel discursivo desde otros sectores diversos de la población.

Desde nuestra perspectiva, la enunciación del principio de no discriminación y el requisito de pluralidad autorizan una interpretación más amplia del derecho a ser informado en la que no sólo se consideren los aspectos formales, sino también la necesidad de contenidos diferenciados. La información plural no sólo debe contemplar la diversidad de sujetos destinatarios que requieren medidas de nivelación en su dimensión formal, sino que debe anticipar a sujetos que desde realidades diversas requieren información diferenciada. Esto último resulta especialmente relevante para aquellos grupos sociales que viven en una 
situación estructural de desventaja a quienes en este trabajo denominamos como grupos susceptibles de discriminación.

\subsection{Igualdad, no discriminación y el derecho a ser informado}

El principio de igualdad y no discriminación se sostienen en el Artículo 7 de la Declaración Universal de los Derechos Humanos de 1948 y se encuentra enunciado explícitamente en el párrafo cuarto del Artículo 1 de la CPEUM reformado en 2011, donde se establece lo siguiente:

Queda prohibida toda discriminación motivada por origen étnico o nacional, el género, la edad, las discapacidades, la condición social, las condiciones de salud, la religión, las opiniones, las preferencias sexuales, el estado civil o cualquier otra que atente contra la dignidad humana y tenga por objeto anular o menoscabar los derechos y libertades de las personas.

Como se muestra en el texto constitucional la prohibición explícita de la discriminación tiene como finalidad garantizar las condiciones que permitan un proyecto de vida digna mediante el combate a aquellos comportamientos que impidan la materialización de los derechos y las libertades de las personas. Sin embargo, cabe preguntarse más ampliamente qué es la discriminación.

Rodríguez Zepeda, uno de los estudiosos más relevantes del fenómeno discriminatorio en México, ha proporcionado la siguiente definición:

La discriminación es una conducta culturalmente fundada y sistemática y socialmente extendida de desprecio contra una persona o grupo de personas sobre la base de un prejuicio negativo o un estigma relacionado con una desventaja inmerecida, y que tiene por efecto (intencional o no) dañar sus derechos y libertades fundamentales (2005: 28).

Como se subraya en esta definición, el hecho de que la discriminación sea un fenómeno "culturalmente fundado" permite comprender que existen grupos sociales más susceptibles de ser discriminados que otros. En la medida en que históricamente ciertas características como el origen étnico, el género, las preferencias sexuales, algunas condiciones de salud, la edad o la condición social han sido consideradas como negativas o inferiores en una sociedad 
con base en prejuicios, estigmas y estereotipos, las personas a las que se les atribuyan estas características se encontrarán en mayor desventaja que las demás.

Así, en México el Consejo Nacional para la Prevención de la Discriminación (CONAPRED) habla de "grupos en situación de discriminación" e identifican once categorías: 1) Adultos mayores, 2) Afrodescendientes; 3) Personas con creencias religiosas no hegemónicas; 4) Personas de determinado origen étnico; 5) Migrantes y refugiados; 6) Mujeres; 7) Niñas y niños; 8) Personas que viven con VIH; 9) Diversidad sexual; 10) Jóvenes; 11) Trabajadoras del hogar.

Tanto en la definición proporcionada por Rodríguez Zepeda, como en el texto constitucional se especifica que la discriminación no sólo tiene por efecto una ofensa personal sino la restricción o limitación de derechos. En estricto sentido se habla de discriminación cuando las conductas hacia terceros basadas en prejuicios y estigmas dificultan la materialización de un derecho concreto. De tal forma que, los grupos arriba mencionados, además de lidiar con los prejuicios y estigmas injustificados, son grupos que sistemáticamente encuentran restricciones y obstáculos para la garantía de sus derechos y libertades fundamentales.

Es por ello que para combatir la discriminación el Estado no sólo está obligado a tratar en igualdad de condiciones a todas las personas, sino a corregir las desigualdades históricas. Por lo mismo, debe buscar todas las herramientas a su alcance para garantizar los derechos si no desea perpetuar estructuras discriminatorias. Esto también vale para el derecho a ser informado: el Estado debe valerse de todos los medios a su alcance para proporcionar la información necesaria a todos los grupos sociales, considerando los formatos pertinentes, pero también los contenidos adecuados.

No obstante, si la valoración del cumplimiento de las exigencias mejor conocidas del derecho a ser informado, como la oportunidad, la veracidad, la verificabilidad y la confiabilidad, resultan difíciles de evaluar, el análisis de la pertinencia de los contenidos informativos diferenciados para grupos sociales susceptibles de discriminación es aún un terreno fértil de estudio. La pertinencia, siguiendo los criterios de las ciencias de la información, indica la medida en la que una fuente, documento o mensaje se ajusta a una necesidad (Korfhage, 1997). 
En ese sentido, el estudio que a continuación presentamos tiene como finalidad identificar los avances y limitaciones experimentadas al intentar incorporar información diferenciada que ayude a estos grupos a tomar decisiones en el contexto de la pandemia por SARS-CoV-2, a la población en general a evitar conductas discriminatorias y a los actores institucionales a erradicarlas. Más específicamente, analizamos si la información brindada cumple al menos con los requisitos mínimos que podrían "ajustarse a las necesidades" de los grupos susceptibles de discriminación al contemplar los riesgos y obstáculos que los aquejan.

\section{El derecho a la información de grupos susceptibles de discriminación en la estrategia de comunicación social de la Secretaria de Salud del Gobierno Federal}

\subsection{Metodología de Análisis}

Para el propósito de este texto, se realizó un análisis de contenido de las conferencias dictadas por el Subsecretario de Prevención y Promoción de la Salud de México, el Dr. Hugo López Gatell. El análisis de contenido permite construir categorías en las que diferentes componentes del mensaje emitido en un discurso son clasificados, con base en una serie de criterios de carácter teórico y/o empírico para posteriormente ser sometidos a un tratamiento de tipo estadístico.

En el caso del presente proyecto de investigación, se revisaron 42 conferencias y del total de los mensajes contenidos en ellas se seleccionaron aquellos que hacían referencia a los grupos susceptibles de discriminación. El contenido de dichos mensajes se clasificó en las siguientes categorías: a) el número de menciones por grupo susceptible de discriminación en el contexto de la pandemia, b) el tipo de información asociada a los riesgos específicos del grupo susceptibles de discriminación y c) el origen de la información. Mediante estas variables se construyó un IPI. A continuación, explicamos las características de cada una de las variables consideradas y posteriormente presentamos los resultados obtenidos.

\section{a) Grupos susceptibles de discriminación en el contexto de la pandemia}

La variable "grupos susceptibles de discriminación en el contexto de la pandemia" amplía la clasificación de CONAPRED e incorpora a grupos sociales que aun sin estar estructuralmente en una "situación" de discriminación pueden ser "susceptibles" de ser 
discriminados en el contexto de la pandemia, como ha ocurrido específicamente con el personal médico debido tanto a su situación laboral como a los prejuicios y estigmas de la población.

Dado que la discriminación está asociada a la negación de derechos, los "grupos susceptibles de discriminación" son aquellos que encuentran riesgos y obstáculos continuos que impiden la materialización de sus derechos. En la siguiente tabla se enlistan los 16 grupos susceptibles de discriminación junto con el tipo de riesgos y obstáculos a los que se enfrentan y que permiten identificarlos como tales en el contexto de esta pandemia (véase Tabla 1).

Tabla 1

Criterios para identificar grupos susceptibles de discriminación que requieren información diferenciada

\section{Grupos susceptibles de discriminación que requiere información diferenciada \\ Riesgos y obstáculos que impiden la materialización de derechos}

Adultos Mayores.

Riesgos asociados a la salud/ Dificultades de movilidad/ Dificultades asociadas a prejuicios y estigmas sociales.

Comunidades rurales/ Barrios, pueblos y Dificultades de infraestructura/ Dificultades comunidades indígenas. asociadas a la diversidad linguística $y$ cultural/ Dificultades asociadas a prejuicios $\mathrm{y}$ estigmas sociales.

Niñas, niños y adolescentes.

Dificultades asociadas a prejuicios $\mathrm{y}$ estigmas sociales/ Dificultades de movilidad/ Violencia.

Migrantes, refugiados y desplazados.

Riesgos asociados a la violencia/ Riesgos asociados a un ambiente insalubre/ Riesgos asociados a las condiciones de la vivienda o a su ausencia.

Mujeres.

Dificultades asociadas a prejuicios y estigmas sociales/ Violencia/ Riesgos asociados al trabajo (doméstico y de cuidados). 
Mujeres embarazadas.

Comunidad LGTTTB+.

Trabajadoras del hogar.

Personas en situación de trata y explotación sexual ${ }^{5}$.

Personas con precariedad laboral.

Personas con discapacidad.

Personas en situación de pobreza.

Personas en situación de calle.

Personas privadas de la libertad.

Personas que viven con VIH.
Riesgos asociados a la salud/ Dificultades asociadas a prejuicios y estigmas sociales.

Dificultades asociadas a prejuicios y estigmas sociales.

Riesgos asociados al trabajo/ Dificultades asociados a prejuicios y estigmas sociales.

Riesgos asociados al trabajo/ Riesgos asociados a la violencia/ Dificultades asociadas a prejuicios y estigmas sociales.

Riesgos asociados al trabajo.

Riesgos asociados a la salud/ Dificultades asociadas a prejuicios y estigmas sociales/ Dificultades asociadas a la movilidad/ Dificultades asociadas a la infraestructura.

Riesgos asociados al trabajo/ Riesgos asociados a las condiciones de la vivienda.

Riesgos asociados a un ambiente insalubre/ Riesgos asociados al trabajo/ Riesgos asociados a la ausencia de vivienda/ Dificultades asociadas a prejuicios $\mathrm{y}$ estigmas sociales).

Riesgos asociados a la violencia/ Riesgos asociados a un ambiente insalubre/ Dificultades de movilidad.

Dificultades asociadas a prejuicios y estigmas sociales/ Riesgos asociados a la salud.

\footnotetext{
${ }^{5}$ Para este texto, se retoman los términos de la Ley general para Prevenir, Sancionar y Erradicar los Delitos en Materia de Trata de Personas, que establece el marco para la garantía de los derechos de las personas en situación de prostitución. No obstante, se debe señalar que existe un debate feminista respecto a la forma de definir, analizar y atender a las personas en situación de prostitución, entre posturas que buscan abolirlo por considerarlo una forma de explotación sexual (con el concepto de trata para fines de explotación sexual) y posturas que pugnan el reconocimiento de la misma como un trabajo (bajo el término de trabajo sexual).
} 
Bernal Lugo, R.; Pedraza Bucio, C. I.; Jiménez Loza, L.; Valtierra Zamudio, J.

Personas con problemas de salud mental. Riesgos asociados a la salud (mental)/dificultades asociadas a prejuicios y estigmas sociales.

Personal de salud.

Dificultades asociadas a prejuicios y estigmas sociales/ Riesgos asociados al trabajo.

Esta clasificación nos permitió identificar la frecuencia con la que estos grupos eran mencionados en la conferencia vespertina de la Secretaria de Salud. Al mismo tiempo, la identificación de los riesgos y obstáculos asociados a cada grupo nos sirvió como criterio para construir la segunda variable tal como se verá a continuación.

b) Tipo de información asociada a los riesgos específicos de los grupos susceptibles de discriminación

A lo largo del texto hemos argumentado que el derecho a ser informado requiere información diferenciada en caso de que esta sea necesaria para la materialización de los derechos. Con base en esta idea, hemos distinguido dos tipos de información de acuerdo al tipo de mención de alguno de estos grupos, a saber: información focalizada o información general (véase Tabla 2).

Tabla 2:

Tipo de información relacionada con los grupos mencionados.

\begin{tabular}{ll}
\hline Tipo de información & Descripción \\
\hline Información focalizada & $\begin{array}{l}\text { Mención de los grupos orientada a proveer } \\
\text { información específica en relación con los } \\
\text { riesgos y obstáculos que impiden la } \\
\text { materialización de derechos. }\end{array}$ \\
Información general & $\begin{array}{l}\text { Mención de los grupos orientada a la } \\
\text { visibilización sin proveer información }\end{array}$ \\
& específica sobre los riesgos relacionados \\
con la pandemia.
\end{tabular}


Como puede observarse consideramos como información focalizada aquella en la que, además de mencionar a alguno de los grupos aquí incluidos, se consideran los riesgos y obstáculos específicos que colocan a ese grupo en una situación de desventaja en el contexto de la pandemia tal como se plantea en la Tabla 1.

De entre los dos tipos de información, la segunda tiene mayor peso en este estudio. Esto porque tanto las personas que son susceptibles de ser discriminadas, como el resto de la población y los actores institucionales necesitan información específica para afrontar situaciones de discriminación, para evitar reproducir estas conductas o, en el mejor de los casos, implementar medidas orientadas a erradicarlas.

\section{c) Origen de la información}

Finalmente, hemos establecido una distinción respecto al origen de la información; es decir, si proviene desde el lugar de la autoridad, independientemente de tratarse de un funcionario público o no, o si proviene de la prensa, mediante la intervención de reporteros y reporteras que cubren la conferencia. En el primer caso refleja una intención consciente por parte de la autoridad de hacer presente a un grupo social específico, mientras que en el segundo es consecuencia de un actor diferente, en este caso las personas que ejercen el periodismo. Con base en estos criterios, establecimos un IPI para mostrar la predominancia de ciertos grupos susceptibles a ser discriminados en el discurso de las conferencias, con los resultados que se explican en el siguiente apartado.

\subsection{Estudio y resultados}

En 35 de las 42 sesiones (84\%) se hizo mención de alguno de estos grupos, además de que todos los grupos considerados en nuestro instrumento (véase Tabla 1) fueron mencionados al menos en una ocasión e incluso se mencionaron otros grupos no contemplados, en particular el grupo de personas desparecidas sobre el que se brindó información relevante.

A partir de estas menciones establecimos una clasificación de 18 grupos: los 16 originalmente contemplados y dos categorías nuevas, personas desaparecidas y otros, esta última categoría responde a la ambigüedad con la que fueron señalados algunos grupos que no terminaron de ser bien definidos. 


\subsubsection{Caracterización general}

El total de menciones de grupos susceptibles de discriminación fue de 92. El promedio de grupos mencionados por sesión fue de 3 , aunque se detectó que en la primera mitad del periodo analizado se ubican las sesiones con el mayor número de menciones. Así, en una de las primeras sesiones analizadas se hizo mención de 10 grupos y en una de las sesiones de la mitad del periodo se registraron seis.

Ahora bien, en cuanto al número de grupos mencionados en función del total de sesiones analizadas se encontró que poco más de la mitad de las sesiones (54\%) solo mencionaron un grupo; en cerca de la décima parte de las sesiones (9.7\%) se hizo mención a dos grupos; en poco más de la décima parte de las sesiones (12.9\%) se hizo mención a tres grupos; en poco de una veinteava parte de las sesiones (6.4\%) se hizo mención a cinco grupos; y en poco más de una décima parte (12.9\%) se hizo mención a seis o más grupos.

En lo que respecta al número de menciones por grupo específico, como se puede observar en la Tabla 3 los grupos con el mayor número de menciones fueron el que corresponde a las Personas en situación de pobreza (14\%), Personas con discapacidad (13\%) y Niñas, niños y adolescentes (12\%). Por el contrario, los grupos menos mencionados fueron: Comunidad LGTTTB+, Personas en situación de calle y Personas en situación de trata. En el caso de estos tres grupos cada uno tuvo una mención equivalente al $1 \%$ del total.

Tabla 3

Porcentaje de menciones por grupos registrados

\begin{tabular}{lccccc}
\hline \multicolumn{1}{c}{ Grupo } & Menciones & $\%$ & \multicolumn{1}{c}{ Grupo } & Menciones & $\%$ \\
\hline $\begin{array}{l}\text { Comunidad } \\
\text { LGTTTB+ }\end{array}$ & 1 & 1.1 & Personas desaparecidas & 4 & 4.1 \\
$\begin{array}{l}\text { Personas en situación } \\
\text { de calle }\end{array}$ & 1 & 1.1 & $\begin{array}{l}\text { Personas con } \\
\text { precariedad laboral }\end{array}$ & 5 & 5.1 \\
$\begin{array}{l}\text { Personas en situación } \\
\text { de trata }\end{array}$ & 1 & 1.1 & $\begin{array}{l}\text { Pueblos, barrios } \\
\text { originarios y } \\
\text { comunidades indígenas }\end{array}$ & 5 & 5.1 \\
& & &
\end{tabular}




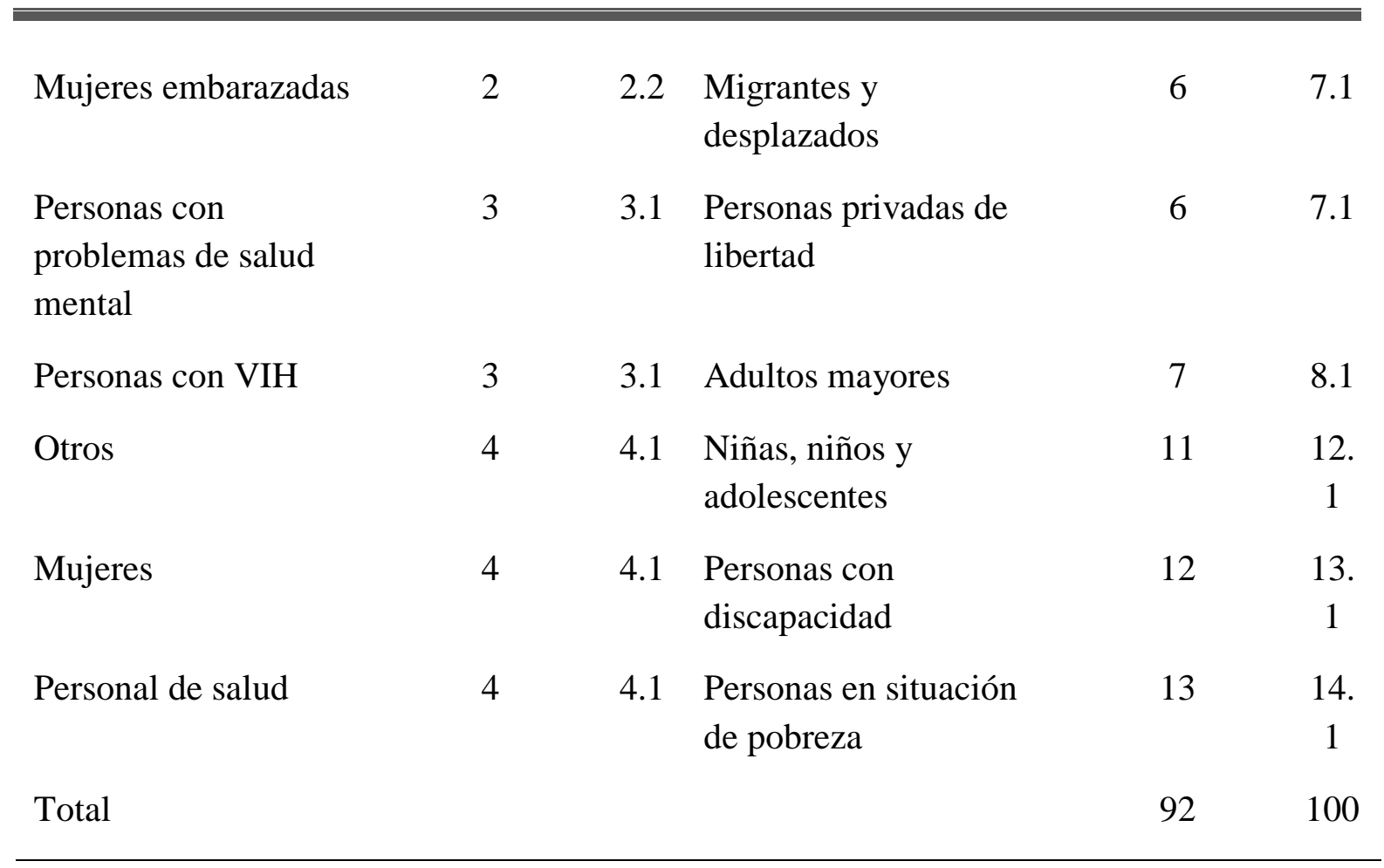

De acuerdo con lo señalado en el apartado teórico del proyecto de investigación, era importante considerar el tipo de información diferenciada que acompañaba la mención de los grupos para así valorar el nivel de reconocimiento por parte de las autoridades. En lo que respecta a este rubro, se encontró que el $66.6 \%$ de la información que se proporcionó a los grupos es de tipo general y sólo 33.3\% fue focalizada. Proporción que se mantuvo en las dos etapas de evolución del programa de atención de la pandemia estructurada por el Gobierno de México.

En cuanto al origen de la información se identificó que todos fueron mencionados al menos una vez por la Autoridad. En este caso se tiene que el $80 \%$ del total de las menciones (74) fueron realizadas por algún funcionario o funcionaria, mientras que el resto (20\%) fueron producto de intervenciones de la prensa. En este sentido, se puede inferir el interés de la prensa por visibilizar a ciertos grupos, aunque este interés no es extensivo a la totalidad de los que fueron mencionados en la conferencia.

\subsection{2. Índice de Prioridad Informativa (IPI)}

En atención al argumento de que el derecho a ser informado requiere de contenidos diferenciados para grupos sociales distintos, especialmente para aquellos que sean 
susceptibles de discriminación, construimos el IPI. Para ello, se calculó un factor que fue el resultado de multiplicar el número de menciones que tuvo el grupo, por el total de sesiones en que fue mencionado, y por un valor de 1 si la información que se refirió durante su mención fue general y 2 si fue específica.

El resultado obtenido se convirtió en la proporción del factor que representa el grupo. Con el puntaje obtenido en el IPI los grupos se clasificaron en prioridad informacional baja, media y alta (véase Tabla 4). Para realizar la clasificación se procedió a estandarizar estadísticamente la variable "Posición”. Así, aquellos grupos cuyo valor de posición se encontraba por debajo de la mediana del IPI fueron clasificados como de baja o muy baja prioridad. Los grupos que se encontraban alrededor de la mediana fueron clasificados como prioridad media y finalmente los grupos que se encontraban por arriba de la mediana fueron clasificados como de prioridad alta o muy alta.

Tabla 4

Índice de Prioridad Informacional (IPI)

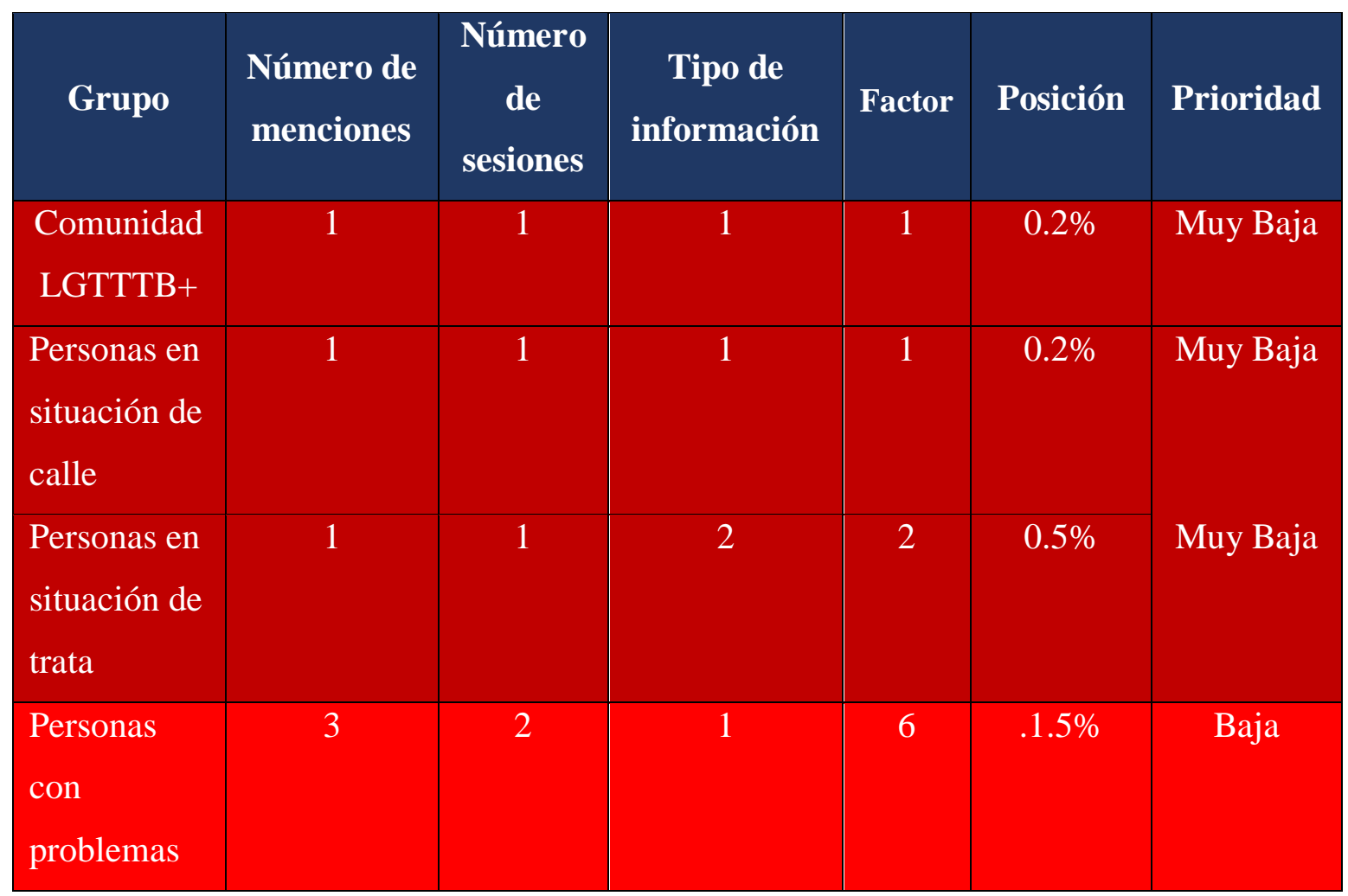



comunicación social del Gobierno Federal mexicano ante la pandemia por SARS-CoV-2

\begin{tabular}{|c|c|c|c|c|c|c|}
\hline $\begin{array}{l}\text { de salud } \\
\text { mental }\end{array}$ & & & & & & \\
\hline $\begin{array}{l}\text { Mujeres } \\
\text { embarazada } \\
\text { S }\end{array}$ & 2 & 2 & 2 & 8 & $2 \%$ & Baja \\
\hline Otros & 4 & 2 & 1 & 8 & $2 \%$ & Baja \\
\hline $\begin{array}{l}\text { Personas } \\
\text { desaparecid } \\
\text { as }\end{array}$ & 4 & 1 & 2 & 8 & $2 \%$ & Baja \\
\hline $\begin{array}{l}\text { Personas } \\
\text { con VIH }\end{array}$ & 3 & 2 & 2 & 12 & $3 \%$ & Baja \\
\hline $\begin{array}{l}\text { Migrantes y } \\
\text { desplazados }\end{array}$ & 6 & 1 & 2 & 12 & $3 \%$ & Baja \\
\hline Mujeres & 4 & 2 & 2 & 16 & $4 \%$ & Media \\
\hline $\begin{array}{l}\text { Personal de } \\
\text { salud }\end{array}$ & 4 & 2 & 2 & 16 & $4 \%$ & Media \\
\hline $\begin{array}{l}\text { Personas } \\
\text { con } \\
\text { precariedad } \\
\text { laboral }\end{array}$ & 5 & 2 & 2 & 20 & $5 \%$ & Media \\
\hline $\begin{array}{l}\text { Pueblos, } \\
\text { barrios } \\
\text { originarios }\end{array}$ & 5 & 2 & 2 & 20 & $5 \%$ & Media \\
\hline $\begin{array}{l}\text { Personas } \\
\text { privadas de } \\
\text { libertad }\end{array}$ & 6 & 2 & 2 & 24 & $6 \%$ & Media \\
\hline $\begin{array}{l}\text { Personas } \\
\text { con } \\
\text { discapacida } \\
\text { d }\end{array}$ & 12 & 2 & 2 & 48 & $12 \%$ & Alta \\
\hline
\end{tabular}




\begin{tabular}{|l|c|c|c|c|c|c|}
\hline $\begin{array}{l}\text { Adultos } \\
\text { mayores }\end{array}$ & 7 & 7 & 1 & 49 & $12 \%$ & Alta \\
\hline $\begin{array}{l}\text { Personas en } \\
\text { situación de } \\
\text { pobreza }\end{array}$ & 13 & 5 & 1 & 65 & $16 \%$ & Alta \\
\hline $\begin{array}{l}\text { Niñas, } \\
\text { niños y } \\
\text { adolescente }\end{array}$ & 11 & 4 & 2 & 88 & $22 \%$ & Muy Alta \\
s & & & & & & \\
\hline
\end{tabular}

Como puede observarse en la Tabla 4 el $16.7 \%$ del total de los grupos identificados se ubicaron en el nivel de prioridad informacional muy baja, el 36.4\% baja, el 24.7\% media, $16.7 \%$ alta y el $5.5 \%$ muy alta. Un dato importante es que en el al interior de cada uno de los niveles también existen jerarquías. Esta distribución de los grupos permite observar la existencia de una estructura jerárquica que coloca a un conjunto de grupos en condiciones de posibilidad de discriminación informacional por debajo de otros.

Así, los grupos que se encuentran en el nivel de dicha posibilidad son, por posición en el IPI: 1) Comunidad LGTTTB+, 2) Personas en situación de calle y 3) Personas en situación de trata. Por el lado contrario, en el nivel más alto de prioridad informacional se encontró el grupo que tiene la mayor posición en el IPI es el de Niñas, niños y adolescentes. Los grupos que le anteceden son: 1) Personas en situación de pobreza, 2) Adultos mayores y 3) Personas con discapacidad.

En cuanto al comportamiento del IPI en las etapas en que se divide el programa de atención a la pandemia se encontró que la totalidad de los grupos que pertenecen al nivel muy bajo de prioridad informacional, cerca del $70 \%$ de los que se encuentran en el nivel bajo y poco más del $40 \%$ de los que se encuentran en el nivel medio fueron visibilizados durante la etapa 2 de la contingencia sanitaria. Por el contrario, la mayor proporción de grupos que tienen prioridad alta y muy alta fueron visibilizados durante la etapa 3 (véase Figura 1). 


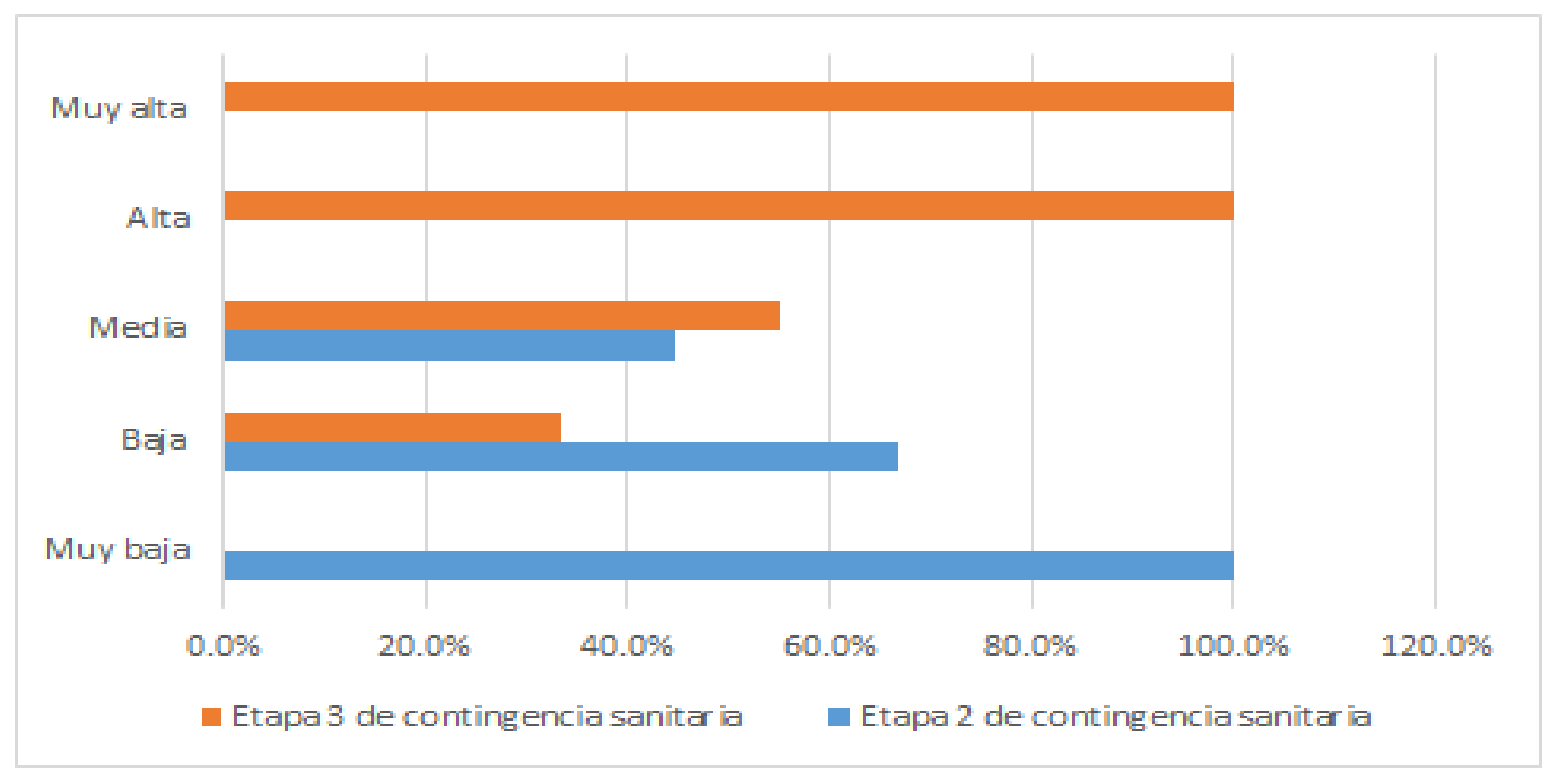

Figura 1: Proporción de grupos visibilizados en las etapas de la Pandemia en función del IPI

Al revisar los criterios que justifican la necesidad de información diferenciada de los grupos con incidencia informativa muy alta, alta y media, encontramos que obstáculos para la movilidad y riesgos de salud aparecen como criterios comunes, en tanto que los grupos con incidencia informativa baja y muy baja comparten los criterios de violencia y prejuicios. Esto permite inferir que aun cuando la intención de la autoridad es visibilizar a los diferentes grupos susceptibles de ser discriminados, se prioriza la información que atiende criterios relacionados con el riesgo de contagio (movilidad y riesgos de salud), colocando en segundo plano los criterios de los riesgos del comportamiento social (violencia y prejuicios).

\section{Conclusiones}

En este artículo hemos presentado los resultados de un estudio sobre las conferencias vespertinas de la Secretaría de Salud en el contexto de la pandemia por SARS-CoV-2 orientado a identificar si en ellas se ofrecen elementos informativos que permitan cumplir los requisitos mínimos para materializar una de las tres dimensiones del derecho a la información, esto es, el derecho a ser informado, de grupos susceptibles de discriminación. Aunque la valoración general de la situación del derecho a la información durante la pandemia implicaría un estudio más amplio que, además de las conferencias vespertinas, incorporara elementos como la difusión de información oficial en medios impresos, 
electrónicos y plataformas digitales por parte del Estado, el papel de la prensa o las participaciones de la autoridad en otros espacios, el estudio de las conferencias vespertinas nos proporciona un acercamiento significativo debido a la relevancia de las mismas en el contexto nacional.

Después de mostrar la importancia del derecho a la información para el ejercicio de la ciudadanía en general y para afrontar las dificultades de la emergencia sanitaria en particular, hemos argumentado que en lo que corresponde a los grupos susceptibles de discriminación se requiere información diferenciada para afrontar los riesgos y obstáculos que impiden la materialización de sus derechos. Esto nos llevó a identificar 16 grupos (Tabla 1) que pueden considerarse como grupos susceptibles de discriminación durante el periodo de emergencia sanitaria, ampliando así la propuesta de 11 "grupos en situación de discriminación" planteada por CONAPRED. Dicha ampliación se justifica porque las circunstancias derivadas de la pandemia generan riesgos y obstáculos particulares, y hacen susceptibles de discriminación a grupos que no necesariamente lo son en otras circunstancias, tal como ocurre con los trabajadores del sector salud. Producto del análisis de las conferencias, hemos identificado dos grupos más: las personas desaparecidas, y un grupo amplio que señalamos con la categoría otros.

A partir de esta clasificación construimos un IPI en el que se toma en cuenta el número de menciones de los grupos, si el tipo de información de las menciones es general o focalizada (tomando como referencia los riesgos y obstáculos específicos de cada grupo) y el origen de la información. Los resultados del estudio de 42 conferencias evidencian que aun cuando existe un interés innegable por parte de las autoridades sanitarias por visibilizar a algunos grupos en situación de desventaja, existen claras diferencias en la atención que se otorga a unos respecto a otros. Así, mientras que grupos como "Niñas, niños y adolescentes", "Personas con discapacidad" y "Personas en situación de pobreza" fueron mencionadas en más de 10 ocasiones, grupos como "Personas en situación de trata", "Personas en situación de calle" y "Comunidad LGTTTB+" sólo fueron mencionados en una ocasión.

Mientras que en el grupo "Comunidad LGTTTB+" la escasa cantidad de menciones podría estar asociada a que desde la perspectiva de la autoridad el único elemento capaz de profundizar las barreras para la materialización de sus derechos en el contexto de la pandemia 
(Dificultades asociadas a prejuicios y estigmas sociales) no sería tan específico como para requerir información diferenciada, pudiendo ser subsumido en los llamados generales a evitar los prejuicios; en el caso de los grupos "Personas en situación de trata" y "Personas en situación de calle" existe una acumulación de riesgos y obstáculos evidentes —en el primer caso se acumulan riesgos asociados al trabajo, riesgos asociados a la violencia y dificultades asociadas a prejuicios y estigmas sociales; mientras que en el segundo se acumulan riesgos asociados a un ambiente insalubre, riesgos asociados al trabajo, riesgos asociados a la ausencia de vivienda y dificultades asociadas a prejuicios y estigmas sociales - que ameritaría mayor atención de la autoridad. En ambos casos se puede afirmar que se trata de omisiones importantes que no permiten la materialización plena del derecho a ser informado.

En lo que corresponde al tipo de información el estudio mostró que sólo el 33\% de la misma fue focalizada mientras que el resto fue "general". Con la única excepción del grupo "Adultos mayores", identificado como un grupo especialmente vulnerable por razones médicas, constatamos que aun en los casos en los que hay un intento de visibilizar a grupos sociales susceptibles de discriminación, como ocurre claramente con los grupos "Personas con discapacidad" y "Personas en situación de pobreza", esto no necesariamente se traduce en la difusión de información específica que permita afrontar las dificultades de la pandemia. Así, a la poca visibilidad de la mayoría de los grupos, debemos añadir la falta de información específica para aquellos que fueron más mencionados.

Debe reconocerse que en la mayoría de las ocasiones (80\%) la incorporación de grupos susceptibles de discriminación se llevó a cabo por parte de la Autoridad, lo que evidencia una elección consciente y un interés por incluir en la agenda a estos grupos, incluso si consideramos las deficiencias recién señaladas. Sin embargo, contrario a lo que suponíamos en el proceso de diseño de nuestros instrumentos metodológicos, la prensa que se encontraba en las conferencias introdujo muy pocas veces a estos grupos en la agenda informativa (20\%), ante lo cual surgen interrogantes que deberán ser estudiadas en otras fases de esta investigación; la más importante de ellas tiene que ver con las razones que explicarían la ausencia de interés hacia estos grupos en la agenda informativa de los medios de comunicación. 
Finalmente, una vez integrados los datos de las tres variables contempladas, el IPI muestra con claridad la existencia de dos tipos de comportamiento respecto a los grupos susceptibles de discriminación que implican dos retos de distinta índole para la materialización plena del derecho a ser informados, aunque en distintas intensidades los grupos considerados en la Tabla 4 como grupos de prioridad muy baja, baja e incluso media son grupos sobre los cuales la omisión de la autoridad abona poco a enfrentar la invisibilización de los riesgos y obstáculos presentes durante la pandemia.

En estos casos, la ausencia de información diferenciada se asocia de manera clara a su marginalidad en el discurso oficial. No obstante, el problema, de los grupos de prioridad alta y muy alta, a excepción del grupo "Adultos mayores", es de distinta naturaleza. A pesar de que la autoridad los coloca deliberadamente en la agenda y reconoce la existencia de desventajas particulares, sólo eventualmente provee de información focalizada que pueda tener algún tipo de efecto en la manera como hacen frente a los retos de la pandemia.

De lo anterior se concluye que: 1) existe un reconocimiento de los grupos susceptibles de discriminación y una intención por parte de la Autoridad de ofrecer información sobre ellos; no obstante, 2) existen diferencias bastante significativas entre los distintos grupos a tal grado que algunos fueron mencionados en más de 10 ocasiones y otros tan sólo en una, por lo que resulta necesario estudiar las razones de este trato desigual; 3) a excepción del grupo adultos mayores, la información ofrecida fue mayoritariamente general y muy pocas ocasiones específica lo que habla de serias dificultades para el cumplimiento de los requisitos mínimos para materializar el derecho a la información de grupos susceptibles de discriminación en el contexto de esta pandemia; 4) aunque la intención de visibilizar a estos grupos en el discurso oficial es un paso importante, es evidentemente insuficiente si se hace de manera desigual y sin ofrecer información pertinente y 5) en la agenda informativa de la prensa que asistió a las conferencias la inclusión de estos grupos no sólo no resulta prioritaria sino que es casi inexistente, lo que lleva a preguntarnos sobre las razones que explican esta circunstancia. 


\section{Declaratoria de disponibilidad de datos.}

Los autores declaran que la información empleada en este documento se obtuvo de las conferencias vespertinas sobre el Informe Diario de Coronavirus Covid19 en México, disponibles en el canal oficial del Gobierno de México:

https://www.youtube.com/channel/UCvzHrtf9by1-UY67SfZse8w

\section{Referencias}

Almenara, J. C. (2008). TICs para la igualdad: la brecha digital en la discapacidad. ANALES de la Universidad Metropolitana 8(2), 15-43.

Carr, N. (2011). Superficiales. ¿Qué está haciendo internet con nuestras mentes? Madrid: Taurus.

Castells, M. (1999). La era de la información. Economía sociedad y cultura. México: Siglo XXI.

Chomsky, N. (2003). La propaganda y la opinión pública. Barcelona: Crítica.

Colmex. (2018). Desigualdades en México 2018. México: Colmex.

Eco, U. (1965). Apocalípticos e integrados. Barcelona: Lumen.

CONAPRED. (2017). Encuesta Nacional sobre Discriminación 2017. Recuperado de https://www.inegi.org.mx/programas/enadis/2017/.

Held, D. (2009). Modelos de democracias. Madrid: Alianza.

Illades, E. (2018). Fake News: la nueva realidad. México: Grijalbo

Korfhage, R. R. (1997). Information storage and retrieval. John Wiley \& Sons, Inc..

López Ayllon, S. (2000). El Derecho a la información como Derecho Fundamental. En Carpizo Jorge y Carbonell, M. (2000). Derecho a la información y derechos humanos, (157-189). México: UNAM.

McIntyre, L. (2019). Posverdad. Madrid: Catedra.

Rodríguez Zepeda, J. (2005), Definición y concepto de la no discriminación. El Cotidiana. 134, 23-29.

Ramonet, I. (1998). Internet el mundo que llega. Madrid: Alianza. 
Bernal Lugo, R.; Pedraza Bucio, C. I.; Jiménez Loza, L.; Valtierra Zamudio, J.

Serrano, P. (2013). La comunicación jibarizada. Barcelona: Península.

Villanueva, E. (2006). Derecho de la información. México: Editorial Miguel Ángel Porrúa. 